\title{
Um jogo de gestão de caos para aprendizagem informal
}

\author{
Bruno Mendes de Souza ${ }^{a}$, Rui Pedro S. de Castro Lopes ${ }^{b}$, Marcos Silvano Almeida ${ }^{a}$ \\ ${ }^{a}$ Universidade Tecnológica Federal do Paraná, Campo Mourão - Paraná, Brasil, ' Instituto Politécnico \\ de Bragança - Bragança, Portugal.
}

\section{Resumo}

A aprendizagem ao longo da vida implica que as pessoas estejam disponíveis para alterar a atitude, a forma de pensar ou de agir, normalmente com base em algum objetivo. Seja com apoio empresarial, devido a requisito de empresa para formação ou informação, seja em ambiente letivo, a aprendizagem implica um contacto com conteúdos e metodologias que estimulam o desenvolvimento pessoal. Muitas vezes, a aprendizagem é estimulada por passagem de documentação, formação presencial ou a distância, b-learning, etc. Neste trabalho, pretende-se avaliar uma forma de aprendizagem informal com base em jogos. A aprendizagem com base em jogos permite experimentar diferentes papéis, correr riscos, errar e repetir sem receio, encorajando o aprendente a contactar e a experimentar os conteúdos. Assim, descreve-se o desenho de um jogo sério com foco na aprendizagem e tendo como principal forma de jogabilidade a gestão e tomada de decisões. $O$ jogo foi desenvolvido para dispositivos iOS e Android, utilizando a ferramenta Unity.

A premissa do jogo coloca o jogador no papel de um gestor empresarial em que é lhe são colocadas diferentes situações que requerem uma decisão. $O$ jogo apresenta uma mecânica baseada em cartas, fazendo com que o jogador escolha entre duas opções, deslizando a carta para direita ou para a esquerda, influenciando as finanças e reputação da empresa. Adicionalmente, o jogo prevê, também, conquistas e cartas colecionáveis, obtidas ao longo do jogo.

O jogo permite que sejam criados diferentes decks para diferentes conteúdos. No momento, foi criado um deck com foco na aprendizagem em cibersegurança e está sendo validado em quatro contextos diferentes. Por meio de questionários, exames antes e após a utilização do jogo e dados recolhidos por observação, é possível obter resultados da satisfação dos alunos e do impacto da utilização do jogo como contributo no processo de aprendizagem. 
Palavras-Chave: Aprendizagem informal, jogos sérios, aprendizagem baseado em jogos.

\section{Introdução}

O termo aprendizagem informal, de uma forma geral, pode ser definido como o aprendizado obtido fora de classes organizadas e estruturadas (McCartney et al., 2011). Além desta definição geral, a aprendizagem informal pode contemplar vários tipos diferentes de aprendizado, como o aprendizado autodirigido, o aprendizado em ambientes informais e o aprendizado no local de trabalho (McCartney et al., 2011). A aprendizagem resultante da realização de atividades diárias e relacionadas a família, trabalho ou lazer (como jogos) também podem ser definidas como uma aprendizagem informal (European Commission, 2001).

A eficácia da aprendizagem pode ser aumentada por intermédio da motivação e do interesse pelo assunto, recorrendo a jogos (Protopsaltis, Pannese, Pappa, \& Hetzner, 2011). Neste sentido, jogos sérios é o termo utilizado para se referir a jogos com intenções além do entretenimento (Deterding, Dixon, Khaled, \& Nacke, 2011). A aprendizagem informal consiste na realização de atividades planejadas que não são explicitamente designadas para a aprendizagem. Assim, os jogos sérios explicitamente projetados para a aprendizagem, se bem planejados e a aprendizagem ocorrer como uma consequência do jogo, podem ser categorizados como experiência de aprendizagem informal (Protopsaltis et al., 2011).

A aprendizagem com base em jogos permite aos jogadores experimentar diferentes papéis, correr riscos, errar e repetir sem receio, encorajando o aprendente a contactar e a experimentar os conteúdos (Pivec, 2007). O objetivo deste trabalho é propor uma forma de aprendizagem informal baseada em jogos, mais especificamente um jogo sério com foco na aprendizagem e tendo como principal forma de jogabilidade a gestão e tomada de decisões. Este jogo tem como foco dispositivos móveis com os sistemas operacionais Android e iOS.

\section{Jogos sérios para dispositivos móveis}

Em geral, os estudantes gostam de jogar e, geralmente jogarão constantemente (Kalloo, Kinshuk, \& Mohan, 2010). Os professores têm dado atenção à utilização de jogos como forma de aprendizagem e como estes podem contribuir para melhorar e facilitar os processos de aprendizagem (Yue \& Ying, 2017). Algumas características devem ser 
estabelecidas no projeto de um jogo sério, como a mecânica. A mecânica é um elemento implementado de forma estratégica pelos desenvolvedores para uma experiência lúdica (mecânicas de jogo) ou atividade de aprendizagem (mecânicas de aprendizagem) (Patino, Romero, \& Proulx, 2016).

A utilização de dispositivos móveis, como smartphones e tablets, é facilitada por alguns aspectos, como a conectividade, pois através do acesso à internet com telemóveis é possível ter uma ampla forma de comunicação e acesso a informações, e recursos, como textos, sons, imagens e vídeos (Fonseca, 2013). Os aspectos de mobilidade e portabilidade permitem ainda que os usuários levem os dispositivos móveis para qualquer lugar e possam utilizar a qualquer momento (Hamid \& Fung, 2007).

Encontra-se pesquisas com jogos em dispositivos móveis aplicados em diversas áreas, por exemplo, no ensino de matemática, Chang e Yang (2016) apresentam um jogo com conceitos de perímetro, área, superfície da área, volume e capacidade e, nos quais, os resultados ao realizar um exame antes e após a utilização do jogo demonstram um progresso significativo na pontuação média dos estudantes. No ensino de cidadania, Chee, Tan, e Liu (2010) demonstram o jogo Statecraft $X$ aplicado a estudantes de 15 anos. No ensino de história, Yue e Ying (2017) apresentam o jogo History Learning Mobile Game (HLMG), que tem como objetivo ensinar história na educação básica.

Outros contextos também podem se beneficiar dos jogos sérios em dispositivos móveis, como é o caso do ensino de primeiros socorros a indivíduos com Transtornos do Espectro Autista (TEA), em que Urturi, Zorrilla, e Zapirain (2011) desenvolveram um jogo para smartphones e tablets, em que os resultados apontam o jogo como tendo contribuído para enriquecer e aumentar o impacto da educação e terapia.

\section{Reigns e a mecânica de deslizar cartas como decisão}

Neste trabalho, o jogo será direcionado aos dispositivos móveis que, pela falta de um teclado físico, faz com que os desenvolvedores utilizem o toque na tela de diversas formas para interação com o usuário. Uma interação comum é o swipe (deslizar) para direita e para a esquerda, utilizado e popularizado no aplicativo de relacionamento Tinder. Inspirados pelo Tinder, diversos aplicativos utilizam a mesma abordagem como, por exemplo, o jogo Reigns.

O Reigns é um jogo desenvolvido pela Nerial e publicado pela Devolver Digital. Lançado em 2016, conta com suporte para as plataformas Android, iOS, Linux, macOS e Microsoft Windows. O jogo decorre num mundo medieval fictício, onde o jogador assume o papel de 
um monarca e governa um reino tomando decisões. $\mathrm{O}$ objetivo é governar pelo maior tempo possível sem desequilibrar os pilares da sociedade: o clero, o povo, o exército e as finanças. Caso não consiga manter o equilíbrio, o rei é morto e um novo rei começa a governar, assim o jogador a cada novo rei tenta conquistar novos objetivos.

A principal forma de jogabilidade utilizada no Reigns é a tomada de decisões deslizando cartas para direita e para esquerda (Fig. 1). As cartas são exibidas de forma aleatória ao jogador, apresentando uma situação em que deve tomar uma decisão. Cada carta é composta de um personagem, um texto e duas opções para escolha. A cada escolha realizada pelo jogador, alterações ocorrem nos recursos como consequências, aumentando ou diminuindo os pilares do seu reino.

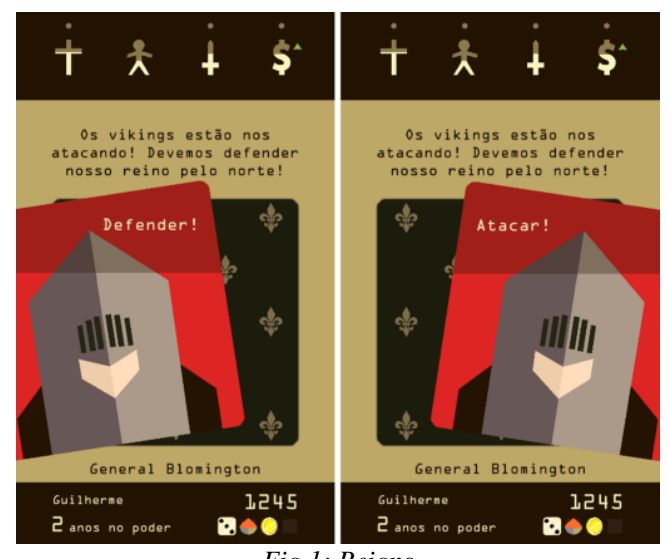

Fig 1: Reigns

Após o lançamento do Reigns, outros jogos também seguiram a mesma linha de jogabilidade, por exemplo o Lapse: A Forgotten Future, o Nirvana: Game of Life e o Soccer Kings. A principal mecânica utilizada na proposta de jogo neste trabalho baseia-se nesta mesma jogabilidade, cartas com situações apresentadas ao jogador e, no qual, deve deslizar para direita ou para esquerda como forma de tomada de decisão.

\section{Proposta de jogo}

O jogo deste trabalho coloca o jogador no papel de um gestor empresarial e, no qual, deve tomar decisões e conciliar para que as finanças e a reputação com a comunidade de sua empresa não cheguem a zero. As situações apresentadas são contextualizadas de acordo com o conteúdo da aprendizagem, pelo que se o conteúdo é sobre cibersegurança, as situações apresentadas para as tomadas de decisões serão sobre o mesmo tema. 
Sempre que o jogo começa inicia-se no dia 0 e, a cada decisão tomada, um novo dia surge. Se acontecer um fim de jogo, por ter ficado sem dinheiro ou reputação perante a comunidade, o jogo é reiniciado, voltando ao dia 0 .

Como citado anteriormente, a forma de interação com o jogo é por meio de cartas em que cada carta apresenta uma situação ao jogador para que seja tomada uma decisão. Uma coleção de cartas, ou seja, um deck, compõem um conteúdo a ser aprendido pelo jogador. $\mathrm{O}$ jogo permite a criação de vários decks com conteúdos diferentes e, então, o jogador escolhe um para ser jogado, permitindo a troca de decks ao decorrer do jogo. O deck, além do conteúdo, também define o enredo, por exemplo, a área de atuação da empresa a ser gerida. $\mathrm{O}$ deck também define outros elementos, como os personagens, conquistas e as cartas colecionáveis (Fig. 2). A tela principal do jogo prevê dois botões, um para a troca de decks e outro para aceder à lista de conquistas. Adicionalmente, também é possível observar a percentagem referente à reputação perante a comunidade, o dia atual, as finanças em euros e, no centro, a carta atual com um texto na parte superior.

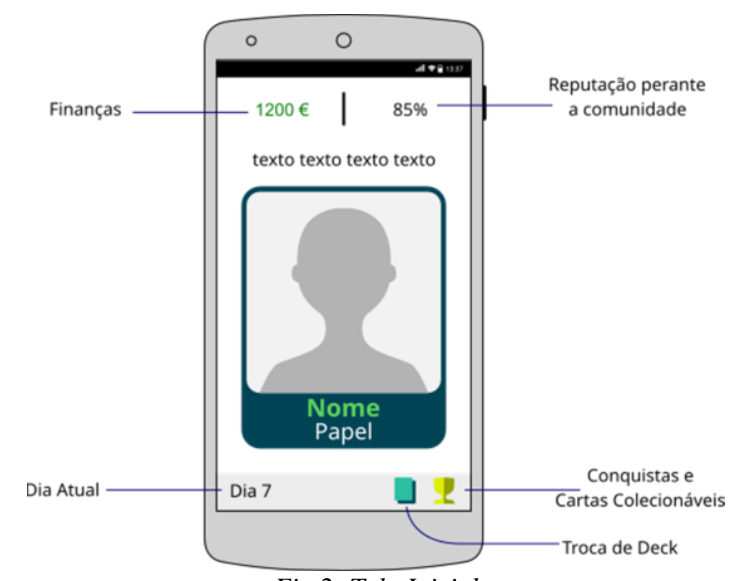

Fig 2: Tela Inicial

Um exemplo de uma jogada é exibido na Fig. 3, no qual a carta com o colaborador Mateus apresenta um questionamento sobre o destino da sobra de tintas. Se o jogador deslizar para a direita tem a opção de investir na reutilização. Se deslizar para esquerda, tem a opção de descartar no rio. Em algumas ocasiões, como neste exemplo, sem ter o real conhecimento das consequências, o jogador pode pressupor que ao investir ele terá um gasto, e ao descartar no rio, uma queda na reputação com a comunidade.

As conquistas e cartas colecionáveis são objetivos extras a serem conquistados pelo jogador ao decorrer do jogo, mantidos mesmo se o jogador perder. As cartas colecionáveis são cartas que não apresentam uma situação de escolha no contexto do deck, que podem representar recursos, benefícios ou vantagens. Atualmente as cartas colecionáveis 
comportam-se apenas como colecionáveis, mas permitem explorar alguns potenciais no futuro.
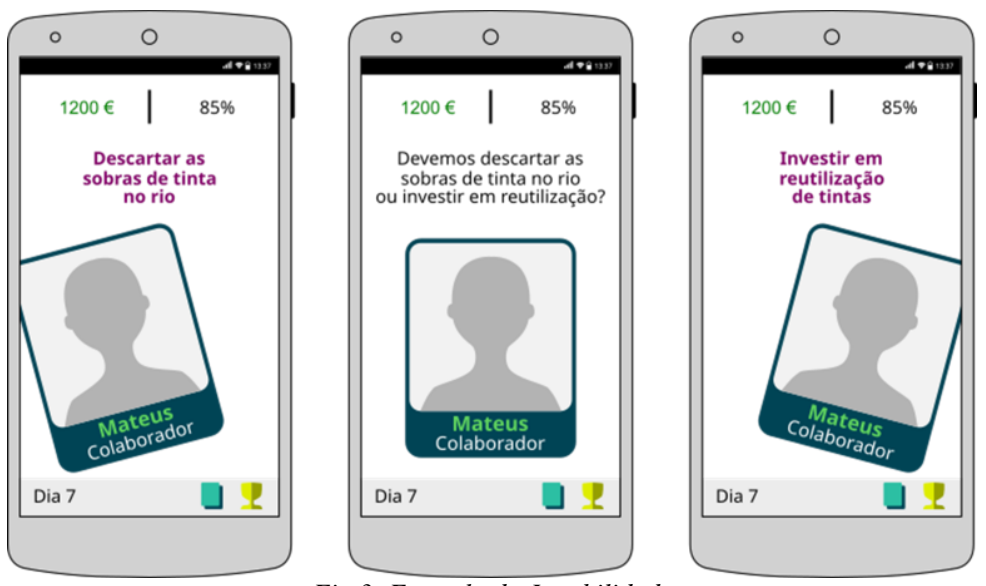

Fig 3: Exemplo da Jogabilidade

As conquistas são objetivos definidos a serem obtidos ao decorrer do jogo, no qual podem ser dias passados no jogo, quantidade de cartas colecionáveis obtidas ou escolhas realizadas. Exemplificando, uma conquista pode ser obtida se o jogador alcançar 30 dias no jogo sem perder ou obter 3 cartas colecionáveis. As conquistas de acordo com escolha realizadas exigem mais percepção do jogador, por exemplo, uma conquista no qual ele deve investir na reutilização de tintas, sendo que o jogador deve estar atento quando vai ter disponível esta decisão ao decorrer do jogo. Na Fig. 4 são exibidas as telas de troca de decks e de objetivos extras, as conquistas e cartas colecionáveis.

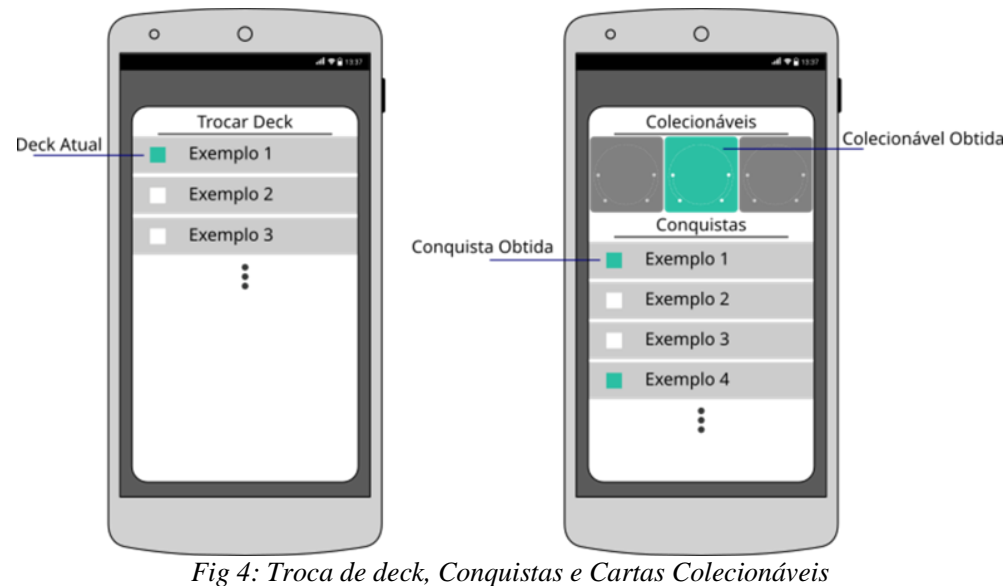

A dinâmica do jogo ocorre da seguinte forma, um conjunto de cartas do deck escolhido pelo 
jogador estarão disponíveis ao decorrer do jogo e, tais cartas surgem de forma aleatória para o jogador. O software deve, para cada jogada, escolha aleatoriamente uma carta a ser apresentada, e após o jogador tomar sua decisão, deve ser aplicado as consequências de sua escolha, e a carta será marcada como exibida. Cartas que já foram exibidas só voltaram a repetir se não houver mais cartas novas a serem apresentadas.

\section{Conclusões e Trabalhos futuros}

O jogo proposto pode permitir a aprendizagem de diferentes conteúdos, como trabalhos futuros se aplica a criação de decks em diferentes contextos e a realização de avaliações e testes a fim de verificar o impactado no aprendizado. No momento, avaliações com alunos do Instituto Politécnico de Bragança estão sendo realizadas com conteúdos de cibersegurança.

A proposta deste trabalho ainda há muitos aspectos para serem explorados. Com a adição de novos elementos e realização de testes avaliações, o jogo pode se tornar uma útil ferramenta para o aprendizado multidisciplinar.

\section{Referências}

Chang, R.-C., \& Yang, C.-Y. (2016). Developing a mobile app for game-based learning in middle school mathematics course. 2016 International Conference on Applied System Innovation (ICASI), 1-2. https://doi.org/10.1109/ICASI.2016.7539807

Chee, Y. S., Tan, E. M., \& Liu, Q. (2010). Statecraft X: Enacting Citizenship Education Using a Mobile Learning Game Played on Apple iPhones. 2010 6th IEEE International Conference on Wireless, Mobile, and Ubiquitous Technologies in Education, 222-224. https://doi.org/10.1109/WMUTE.2010.16

de Urturi, Z. S., Zorrilla, A. M., \& Zapirain, B. G. (2011). Serious Game based on first aid education for individuals with Autism Spectrum Disorder (ASD) using android mobile devices. 2011 16th International Conference on Computer Games (CGAMES), 223-227. https://doi.org/10.1109/CGAMES.2011.6000343

Deterding, S., Dixon, D., Khaled, R., \& Nacke, L. (2011). From Game Design Elements to Gamefulness: Defining "Gamification." Proceedings of the 15th International Academic MindTrek Conference on Envisioning Future Media Environments - MindTrek '11, 9. https://doi.org/10.1145/2181037.2181040

European Commission. (2001). Making a European area of lifelong learning a reality. Communication from the Commission. $\operatorname{COM}(2001) 678$ final. In European Commission. 
Brussels, Belgium.

Fonseca, A. G. M. F. da. (2013). APRENDIZAGEM, MOBILIDADE E CONVERGÊNCIA: Mobile Learning com Celulares e Smartphones. Revista Eletrônica Do Programa de Pós-Graduação Em Mídia e Cotidiano, 2(2), 265. https://doi.org/10.22409/ppgmc.v2i2.9685

Hamid, S. H. A., \& Fung, L. Y. (2007). Learn Programming by Using Mobile Edutainment Game Approach. 2007 First IEEE International Workshop on Digital Game and Intelligent Toy Enhanced Learning (DIGITEL'07), 170-172. https://doi.org/10.1109/DIGITEL.2007.31

Kalloo, V., Kinshuk, \& Mohan, P. (2010). Personalized game based mobile learning to assist high school students with mathematics. Proceedings - 10th IEEE International Conference on Advanced Learning Technologies, ICALT 2010, 485-487. https://doi.org/10.1109/ICALT.2010.140

McCartney, R., Eckerdal, A., Moström, J. E., Sanders, K., Thomas, L., \& Zander, C. (2011). Computing students learning computing informally. Proceedings of the 10th Koli Calling International Conference on Computing Education Research, 43-48. https://doi.org/10.1145/1930464.1930470

Patino, A., Romero, M., \& Proulx, J. N. (2016). Analysis of game and learning mechanics according to the learning theories. 2016 8th International Conference on Games and Virtual Worlds for Serious Applications, VS-Games 2016, 1-4. https://doi.org/10.1109/VS-GAMES.2016.7590337

Pivec, M. (2007). Editorial: Play and learn: potentials of game-based learning. British Journal of Educational Technology, 38(3), 387-393. https://doi.org/10.1111/j.1467-8535.2007.00722.x

Protopsaltis, A., Pannese, L., Pappa, D., \& Hetzner, S. (2011). Serious Games and Formal and Informal Learning. ELearning Papers, 25(July 2011), 1-10.

Yue, W. S., \& Ying, C. Y. (2017). The Evaluation Study of Gamification Approach in Malaysian History Learning via Mobile Game Application. 2017 IEEE 17th International Conference on Advanced Learning Technologies (ICALT), 150-152. https://doi.org/10.1109/ICALT.2017.69 